\title{
Genes for tRNA recycling are upregulated in response to infection with Theiler's mouse encephalitis virus
}

Mineaki Seki ${ }^{1,{ }^{*}}$, Akihiko Komuro ${ }^{1,2}$, Tatsuya Ishikawa ${ }^{1}$, Masayuki Takahashi ${ }^{1}$, and Masayuki Nashimoto ${ }^{1}$

${ }^{1}$ Research Institute for Healthy Living, Niigata University of Pharmacy and Applied Life

Sciences, Niigata, Niigata, 956-8603, Japan

${ }^{2}$ Faculty of Pharmaceutical Sciences, Department of Biochemistry, Niigata University of Pharmacy and Applied Life Sciences, Niigata, Niigata, 956-8603, Japan

${ }^{*}$ Correspondence: Research Institute for Healthy Living, Niigata University of Pharmacy and Applied Life Sciences, Niigata, Niigata, 956-8603, Japan

Tel: +81-250-28-5330;

Fax: +81-250-25-5021;

e-mail: seki@nupals.ac.jp (M. S.) 


\begin{abstract}
The concept of tRNA recycling has recently emerged from the studies of ribosomeassociated quality control. Therein tRNase ZS removes the 2', 3'>p from the ANKZF1cleaved tRNA and the subsequent TRNT1 action re-generates the intact tRNA. To know the roles of the tRNA recycling in vivo, we investigated how viral infection affects the tRNA recycling system by analyzing the mRNA levels of tRNase ZS and TRNT1. We found that both genes in HeLa cells are upregulated in response to infection of Theiler's mouse encephalitis virus but not to that of an influenza A virus. Upregulation was also observed in cells infected with encephalomyocarditis virus with reduced efficiency. The levels of the IFN$\beta$ mRNA appeared to positively correlate with those of the tRNase ZS and TRNT1 mRNAs. The tRNase ZS gene may be regulated post-transcriptionally in the cells infected with Theiler's mouse encephalitis virus.
\end{abstract}

\title{
Keywords
}

tRNA recycling, tRNase ZS, ELAC1, TRNT1, ribosome quality control, tRNA processing, interferon, infection

\section{Abbreviations}

TMEV, Theiler's mouse encephalitis virus

EMCV, encephalomyocarditis virus

IAV, influenza A virus

IFN- $\beta$, interferon beta

MOI, multiplicity of infection 


\section{Introduction}

tRNA recycling has recently found in the studies of ribosome-associated quality control (RQC). The physiological roles are unclear and biochemical studies revealed that two enzymes, namely tRNase ZS (ELAC1) and TRNT1 are involved in this reaction ${ }^{1,2}$. tRNase ZS is a paralog of tRNA maturation enzymes, tRNase ZL (or ELAC2), which cleaves pretRNAs at the junction of a discriminator nucleotide and a 3' trailer sequence, enabling the subsequent $3^{\prime}$-terminal CCA addition ${ }^{3-5}$. On the other hand, tRNase ZS has very limited pretRNA-cleaving activity compared with tRNase $Z L^{6,7}$. This property together with the fact that the tRNase ZS gene is dispensable for viability of at least one human cell line made us curious about its physiological role ${ }^{8}$. Another tRNA maturation enzyme, TRNT1 (or the CCA-adding enzyme), catalyzes the addition of CCA nucleotides ${ }^{9}$. This activity is used not only for tRNA maturation but for tRNA surveillance and quality control ${ }^{10,11}$.

RQC prevents cells from accumulating faulty proteins that can have cytotoxic properties by degrading the aberrant polypeptides ${ }^{12,13}$. This process starts with ubiquitination of an aberrant nascent chain and ends with its proteasomal degradation after extracting it from a $60 \mathrm{~S}$ ribosome-nascent chain complex (60S-RQC). This extraction relies on the peptidyl-tRNA hydrolase activity of ANKZF1, which can cleave the peptidyl-tRNA in the 60S-RQC at the junction of the 3'-terminal CCA nucleotides and the discriminator nucleotide to release the tRNA with a $2^{\prime}, 3^{\prime}$-cyclic phosphate $\left(2^{\prime}, 3^{\prime}>p\right){ }^{14-16}$. The CCA-less tRNA with the 2', 3'>p seemed to be resistant not only to exonuclease digestion but also to repair by TRNT1. This apparent dead-end situation and the enigma of role of tRNase ZS have been solved simultaneously by the discovery that tRNase ZS can remove the $2^{\prime}, 3^{\prime}>p$ from the ANKZF1-cleaved tRNA and that the subsequent TRNT1 action re-generates the intact tRNA ${ }^{1,2}$.

Here, we investigated how viral infection affects the tRNA recycling system by analyzing the expression levels of tRNase ZS and TRNT1. We uncovered that that both 
genes are upregulated in response to infection of Theiler's mouse encephalitis virus (TMEV) or the encephalomyocarditis virus (EMCV) but not to that of an influenza A virus (IAV).

\section{Materials and methods}

\section{Cell culture}

HeLa cells were cultured in complete DMEM media (DMEM media containing $10 \%$ fetal bovine serum and $100 \mathrm{U} / \mathrm{mL}$ penicillin-streptomycin) at $37{ }^{\circ} \mathrm{C}$ in a $5 \% \mathrm{CO}_{2}$ incubator.

\section{Viral infection}

HeLa cells were seeded and cultured in 24-well plates. After washing with phosphate-buffered saline, the cells were incubated in reduced ( $2 \%$ fetal calf serum) complete media containing virus for $2 \mathrm{~h}$. Subsequently, the media was replaced by normal (10\% fetal calf serum) complete media ${ }^{17}$. The cells were then collected at the indicated time points and total RNA was extracted.

\section{Quantitative reverse transcription PCR (qRT-PCR) analysis}

Total RNA was extracted using the RNAiso Plus (Takara Bio, Shiga, Japan). cDNA synthesis was performed using the ReverTra Ace qPCR RT Master Mix with gDNA Remover (Toyobo, Osaka, Japan) in accordance with the manufacturer's protocol. mRNA levels were quantitated using the THUNDERBIRD SYBR qPCR Mix (Toyobo) and normalized against glyceraldehyde-3-phosphate dehydrogenase (GAPDH) mRNA levels. The fold-change in mRNA levels was calculated using the standard curve method. The primer pairs used are listed in Table 1.

\section{RT-PCR assays}


Total RNA was extracted from IAV-infected cells using the RNAiso Plus kit. cDNA synthesis was performed using the ReverTra Ace qPCR RT Master Mix with gDNA Remover in accordance with the manufacturer's protocol. RT-PCR was performed using the AmpliTaq Gold 360 Master Mix (Thermo Fisher Scientific, Waltham, MA, USA). The reaction mixture $(20 \mu \mathrm{L})$ included the IAV protein M primer pair $(4 \mu \mathrm{M})$ and cDNA template $(6 \mathrm{ng}){ }^{18}$. PCR was performed for 36 cycles, and the products were separated on a $2 \%$ agarose gel.

\section{Immunoblotting}

HeLa cells were suspended in NETN buffer (50 mM Tris- $\mathrm{HCl}$ [pH 7.5], $150 \mathrm{mM} \mathrm{NaCl,}$ $1 \mathrm{mM}$ EDTA, $1 \% \mathrm{NP}-40$, and $14.4 \mu \mathrm{M}$ 2-mercaptoethanol), and incubated with a protease inhibitor cocktail (Roche, Basel, Switzerland) at $4^{\circ} \mathrm{C}$ for $20 \mathrm{~min}$. After centrifugation, the supernatant was mixed with sodium dodecyl sulphate (SDS) loading buffer and boiled for 5 $\min$ at $95^{\circ} \mathrm{C}$. After boiling, the samples were applied to a $4-15 \%$ polyacrylamide gradient/SDS gel (BIO-RAD, Hercules, CA). Proteins were electrophoretically separated and transferred to polyvinylidene fluoride (PVDF) membranes (Immobilon P, Merck Millipore, Burlington, MA, USA). Blocking treatment was performed using the PVDF Blocking Reagent for Can Get Signal (Toyobo) and the membranes were incubated with primary antibodies diluted with the Can Get Signal 1 reagent overnight. After incubation with secondary antibodies diluted with the Can Get Signal 2 reagent, the bands were visualized through chemiluminescence.

\section{Antibodies}

The following antibodies were obtained commercially: anti-tRNase ZS (sc-101113, Santa Cruz, Dallas, TX, USA), anti-TRNT1 (NBP1-86589, Novus Biologicals, CO, USA), and anti-Vinculin (Proteintech, Rosemont, IL, USA).

\section{Viruses}


TMEV (DA strain) and IAV (PR8 strain) were generous gifts from Dr. Yoshiro Ohara (Kanazawa Medical University, Ishikawa, Japan) and Dr. Toshiki Himeda (Kanazawa Medical University), and Dr. Mitsutoshi Yoneyama (Medical Mycology Research Center, Chiba University, Chiba, Japan), respectively. EMCV (VR-129B strain S) was purchased from the American Type Culture Collection (Manassas, VA, USA).

\section{Statistical analysis}

Results were presented as mean \pm standard deviation (SD). Differences between the control cells and the cells infected with EMCV were analyzed by Student t-test.

\section{Results}

tRNase ZS and TRNT1 mRNA levels are upregulated in cells infected with TMEV.

We examined tRNase ZS and TRNT1 mRNA levels in HeLa cells after viral infection using qRT-PCR. HeLa cells were infected with TMEV at two different multiplicities of infection (MOI), normal $\mathrm{MOI}(\mathrm{MOI}=1)$ and high $\mathrm{MOI}(\mathrm{MOI}=10)$, and the mRNA levels were measured 10 and $16 \mathrm{~h}$ after the infection. The tRNase ZS and TRNT1 mRNAs were upregulated up to 13-fold and 3.6-fold, respectively, in $10 \mathrm{~h}$, and neither mRNA was upregulated in $16 \mathrm{~h}$ (Fig. 1A and B). Upregulation of the tRNase ZL mRNA was not observed in either MOI (Fig. 1C). The infection of TMEV was confirmed by the upregulation of the interferon $\beta$ (IFN- $\beta$ ) mRNA (Fig. 1D). The IFN- $\beta$ mRNA was highly upregulated in a MOIdependent manner (by 480- and 2800-fold) in $10 \mathrm{~h}$, and its levels were drastically decreased in $16 \mathrm{~h}$, resulting in reductions to 3 and $10 \%$, respectively (Fig. 1D).

tRNase ZS and TRNT1 mRNA levels are upregulated in EMCV-infected cells with reduced efficiencies. 
Overall, both mRNAs were upregulated in a time- and a MOI-dependent manner in the EMCV-infected HeLa cells, albeit with reduced efficiency. The maximum upregulation of the tRNase ZS mRNA was by 2.7 -fold in $16 \mathrm{~h}$ after the infection with $\mathrm{MOI}=10$ (Fig. 2A), and the TRNT1 mRNA level reached to 2.2-fold in $10 \mathrm{~h}$ after the infection with $\mathrm{MOI}=10$ (Fig. 2B). The tRNase ZL mRNA was not upregulated at all (Fig. $2 \mathrm{C}$ ). The IFN- $\beta$ mRNA was also highly upregulated like in the cells infected with TMEV, although its time- and MOIdependency was not observed (Fig. 2D).

tRNase ZS and TRNT1 mRNA levels are not upregulated in cells infected with IAV.

IAV neither upregulated tRNase ZS and TRNT1 mRNAs nor the tRNase ZL mRNA in the HeLa cells. (Fig. 3A-C). Since upregulation of the IFN- $\beta$ mRNA was rarely observed following the IAV infection (data not shown), most likely due to interference by NS1 protein in IAV ${ }^{19}$, its infection was confirmed through amplification of the mRNA encoding the IAV protein M. The protein M mRNA level in the IAV-infected HeLa cells was increased in a timeand a MOI-dependent manner (Fig. 3D).

tRNase ZS and TRNT1 proteins are upregulated in cells infected with TMEV.

The tRNase ZS and TRNT1 protein levels after infection with TMEV were also examined. Although the expression pattern varied by experiments, on the whole, both protein levels after the $\mathrm{MOI}=1$ infection increased up to $5 \mathrm{~h}$ and then decreased. And in the $\mathrm{MOI}=10$ experiment, the tRNase $\mathrm{ZS}$ level reached a maximum in $3 \mathrm{~h}$ and the TRNT1 level continued increasing. A typical pattern is shown in Fig. 4. 


\section{Discussion}

Cellular responses to viral infection are mostly controlled by IFNs and more than 300 genes have been shown to be upregulated by them ${ }^{20,21}$. Since the level of the IFN- $\beta$ mRNA appeared to positively correlate with those of the tRNase ZS and TRNT1 mRNAs (Fig. 1-3), both genes may also be controlled by IFNs. Genes upregulated via the IFN-dependent pathway possess interferon stimulated responsible elements (ISRE) and/or IFN- $\gamma$ activated site (GAS) elements in the promoter region ${ }^{22}$. Further study from this aspect would elucidate a mechanism for tRNase ZS and TRNT1 gene upregulation under viral infection.

Although the tRNase ZS protein levels were also increased in the TMEV-infected cells, they were much lower than the levels expected from the mRNA levels (Fig. 1A and Fig. 4). This observation suggests that tRNase ZS may be regulated post-transcriptionally in the cells infected with TMEV and is consistent with our previous finding that $t R N a s e$ ZS appears to be controlled post-transcriptionally in HEK293 cells ${ }^{23}$.

As far as we know, this is the first report showing that the mRNA levels of tRNase ZS and TRNT1, both of which are necessary for tRNA recycling, are upregulated by infection with TMEV or EMCV. We are currently trying to address the issues of whether tRNA recycling is indeed augmented under viral infection and, if so, what its physiological significance is.

\section{Conflict of interest}

The authors declare no conflicts of interest.

\section{Acknowledgement}

We thank Arisa Haino for her technical assistance. A research grant from the Niigata University of Pharmacy and Applied Life Sciences was received. The funding source was not involved in this study nor the publication of its results. 


\section{References}

1. Yip MCJ, Savickas S, Gygi SP, Shao S. ELAC1 Repairs tRNAs Cleaved during Ribosome-Associated Quality Control. Cell Rep 2020; 30:2106-2114.e5.

2. Yip MCJ, Keszei AFA, Feng Q, Chu V, McKenna MJ, Shao S. Mechanism for recycling tRNAs on stalled ribosomes. Nat Struct Mol Biol 2019; 26:343-9.

3. Vogel A, Schilling O, Späth B, Marchfelder A. The tRNase Z family of proteins: physiological functions, substrate specificity and structural properties. Biol Chem 2005; 386:1253-64.

4. Nashimoto M. Distribution of both lengths and $5^{\prime}$ terminal nucleotides of mammalian pre-tRNA $3^{\prime}$ trailers reflects properties of 3' processing endoribonuclease. Nucleic Acids Res 1997; 25:1148-54.

5. Schiffer S, Rösch S, Marchfelder A. Assigning a function to a conserved group of proteins: the tRNA 3'-processing enzymes. EMBO J 2002; 21:2769-77.

6. Takaku H, Minagawa A, Takagi M, Nashimoto M. A candidate prostate cancer susceptibility gene encodes tRNA 3' processing endoribonuclease. Nucleic Acids Res 2003; 31:2272-8.

7. Yan $\mathrm{H}$, Zareen $\mathrm{N}$, Levinger L. Naturally occurring mutations in human mitochondrial pretRNASer(UCN) can affect the transfer ribonuclease $Z$ cleavage site, processing kinetics, and substrate secondary structure. J Biol Chem 2006; 281:3926-35.

8. Yanaihara N, Kohno T, Takakura S, Takei K, Otsuka A, Sunaga N, Takahashi M, Yamazaki M, Tashiro H, Fukuzumi Y, et al. Physical and transcriptional map of a 311-kb segment of chromosome 18q21, a candidate lung tumor suppressor locus. Genomics 2001; 72:169-79.

9. Slade A, Kattini R, Campbell C, Holcik M. Diseases Associated with Defects in tRNA CCA Addition. Int J Mol Sci 2020; 21:3780.

10. Kuhn C-D, Wilusz JE, Zheng Y, Beal PA, Joshua-Tor L. On-Enzyme Refolding Permits Small RNA and tRNA Surveillance by the CCA-Adding Enzyme. Cell 2015; 160:644-58.

11. Wilusz JE, Whipple JM, Phizicky EM, Sharp PA. tRNAs Marked with CCACCA Are Targeted for Degradation. Science 2011; 334:817-21.

12. Joazeiro CAP. Mechanisms and functions of ribosome-associated protein quality control. Nat Rev Mol Cell Biol 2019; 20:368-83.

13. Brandman O, Hegde RS. Ribosome-associated protein quality control. Nat Struct Mol Biol 2016; 23:7-15.

14. Verma R, Reichermeier KM, Burroughs AM, Oania RS, Reitsma JM, Aravind L, Deshaies RJ. Vms1 and ANKZF1 peptidyl-tRNA hydrolases release nascent chains from stalled ribosomes. Nature 2018; 557:446-51.

15. Kuroha K, Zinoviev A, Hellen CUT, Pestova TV. Release of Ubiquitinated and Non-ubiquitinated Nascent Chains from Stalled Mammalian Ribosomal Complexes by ANKZF1 and Ptrh1. Mol Cell 2018; 72:286-302.e8. 
16. Zurita Rendón O, Fredrickson EK, Howard CJ, Van Vranken J, Fogarty S, Tolley ND, Kalia R, Osuna BA, Shen PS, Hill CP, et al. Vms1p is a release factor for the ribosome-associated quality control complex. Nat Commun 2018; 9:2197.

17. Komuro A, Homma Y, Negoro T, Barber GN, Horvath CM. The TAR-RNA binding protein is required for immunoresponses triggered by Cardiovirus infection. Biochem Biophys Res Commun 2016; 480:187-93.

18. Tripathi S, Tecle T, Verma A, Crouch E, White M, Hartshorn KL. The human cathelicidin LL-37 inhibits influenza A viruses through a mechanism distinct from that of surfactant protein $D$ or defensins. J Gen Virol 2013; 94:40-9.

19. Wang X, Li M, Zheng H, Muster T, Palese P, Beg AA, García-Sastre A. Influenza A Virus NS1 Protein Prevents Activation of NF-KB and Induction of Alpha/Beta Interferon. J Virol 2000; 74:11566-73.

20. Sadler AJ, Williams BRG. Interferon-inducible antiviral effectors. Nat Rev Immunol 2008; 8:55968.

21. Der SD, Zhou A, Williams BRG, Silverman RH. Identification of genes differentially regulated by interferon, or using oligonucleotide arrays. Proc Natl Acad Sci 1998; 95:15623-8.

22. Michalska A, Blaszczyk K, Wesoly J, Bluyssen HAR. A Positive Feedback Amplifier Circuit That Regulates Interferon (IFN)-Stimulated Gene Expression and Controls Type I and Type II IFN Responses. Front Immunol 2018; 9:1135.

23. Takahashi $\mathrm{M}$, Takaku $\mathrm{H}$, Nashimoto $\mathrm{M}$. Regulation of the human tRNase $\mathrm{Z}^{\mathrm{S}}$ gene expression. FEBS Lett 2008; 582:2532-6. 


\section{Figure legends}

Figure 1. The mRNA levels of tRNase ZS and TRNT1 after infection with TMEV. The tRNase ZS (A), TRNT1 (B), tRNase ZL (C), and IFN- $\beta$ (D) mRNAs were analyzed by qRT-PCR. HeLa cells were infected with TMEV at MOls of 1 and 10. The mRNA levels are normalized against the GAPDH mRNA levels and expressed relative to those in the non-infected cells. Error bars indicate SD $(n=3)$.

Figure 2. The mRNA levels of tRNase ZS and TRNT1 after infection with EMCV. The tRNase ZS (A), TRNT1 (B), tRNase ZL (C), and IFN- $\beta$ (D) mRNAs were analyzed by qRTPCR. HeLa cells were infected with EMCV at MOls of 1 and 10. The mRNA levels are normalized against the GAPDH mRNA levels and expressed relative to those in the noninfected cells. Error bars indicate $S D(n=3) .{ }^{*} P<0.05$ vs. control.

Figure 3. The mRNA levels of tRNase ZS and TRNT1 after infection with IAV. The tRNase ZS (A), TRNT1 (B), tRNase ZL (C), and IFN- $\beta$ (D) mRNAs were analyzed by qRT-PCR. HeLa cells were infected with IAV at MOIs of 0.2 and 1. The mRNA levels are normalized against the GAPDH mRNA levels and expressed relative to those in the non-infected cells. Error bars indicate SD $(n=3)$. (D) RT-PCR products from the IAV protein M mRNA are shown. GAPDH cDNA was used as a loading control.

Figure 4. The protein levels of tRNase ZS and TRNT1 after infection with TMEV. HeLa cells were infected with TMEV at MOls of 1 and 10. Whole-cell extracts were prepared 3, 5, 8, and $16 \mathrm{~h}$ after infection. Vinculin was used as a loading control. 
Table 1. Sequences of primers used in this study.

Primer Sequence (5'-3')

tRNase ZS F GGTGTGAGGCGAGTGCTG

tRNase ZS R AGATTTCAATAGGCTGTTTGGAC

tRNase ZL F GACACACAGCACAACGTCCC

tRNase ZL R GACCTTCATGTGGTCAAAGGCAAC

GAPDH F

CCСАСТССТССАССTTTGAC

GAPDH R

ACCCTGTTGCTGTAGCCAAA

IFN- $\beta$ F

GGAAAACGCATGAGCAGTCT

IFN- $\beta$ R

CTATGGTCCAGGCACAGTGA

IAV Protein M F

AGACCAATCCTGTCACCTCTGA 
Figure 1

(A)

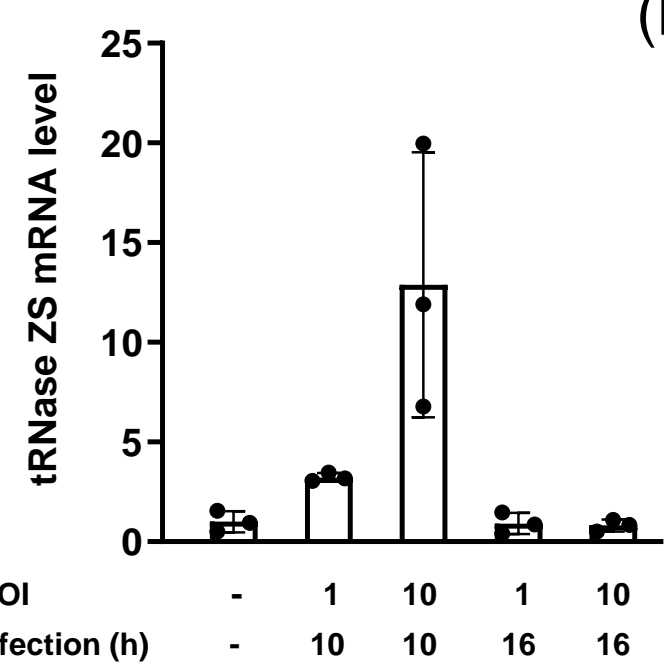

(C)

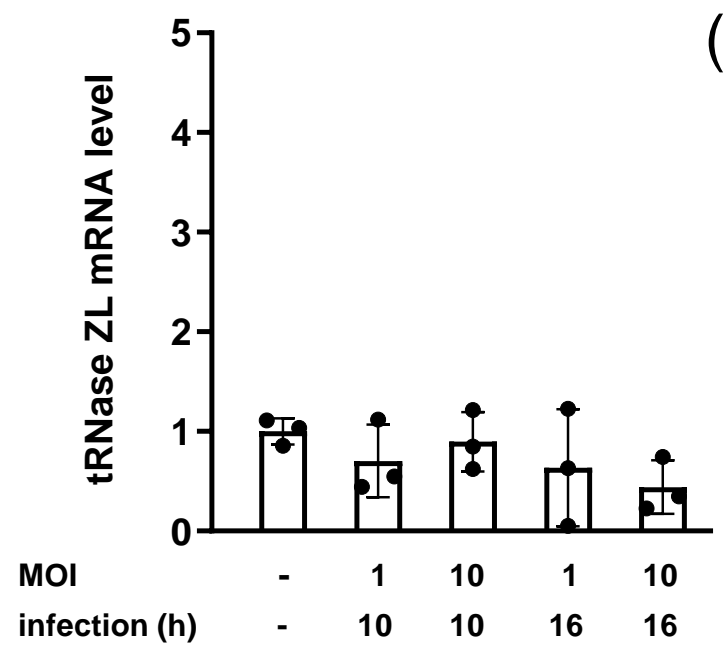

(B)

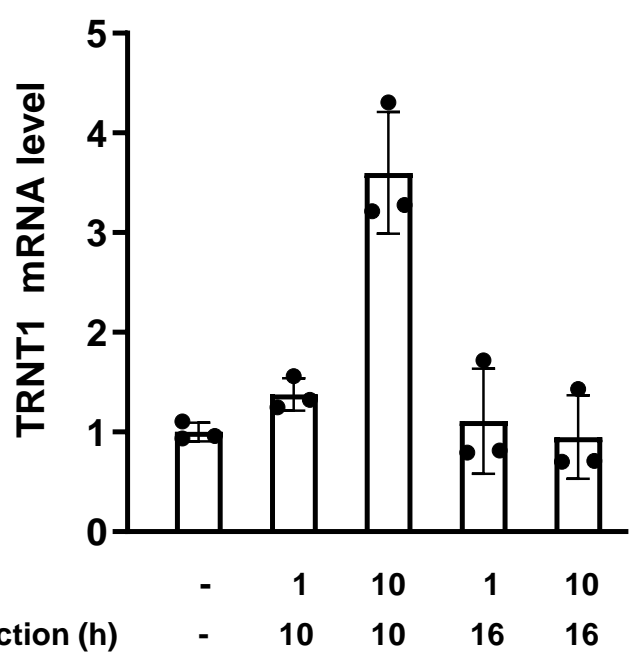

(D)

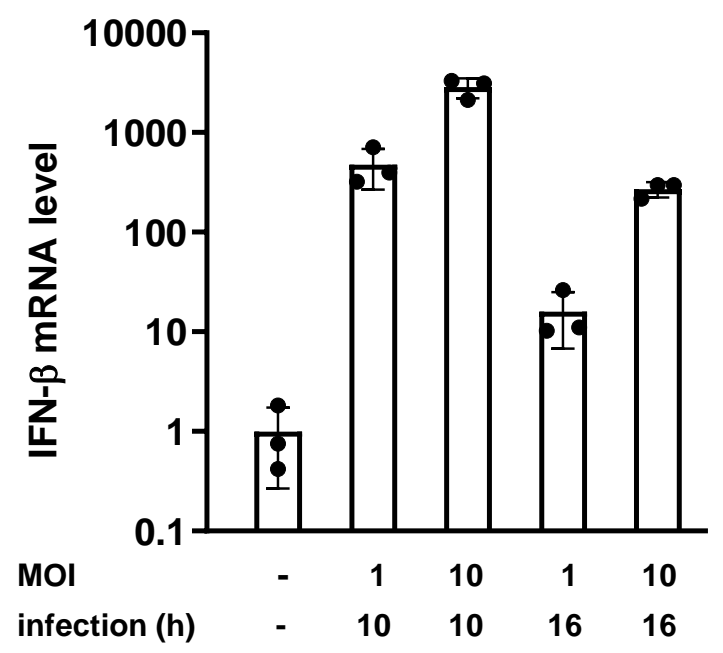


Figure 2

(A)

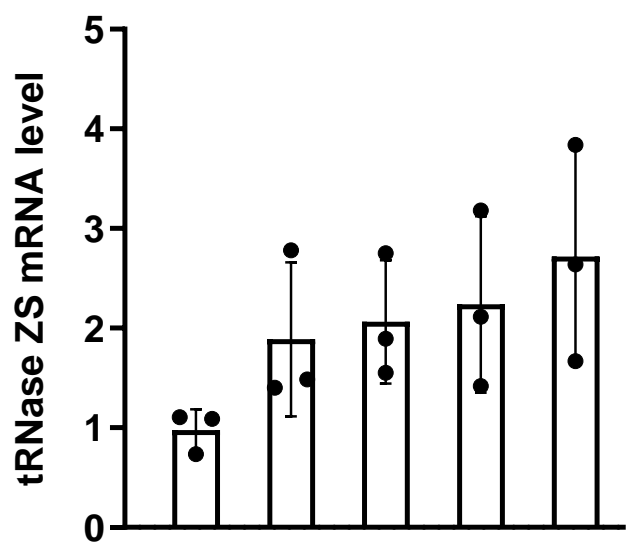

MOI

$\begin{array}{llllll}\text { infection (h) } & - & 10 & 10 & 16 & 16\end{array}$

(C)

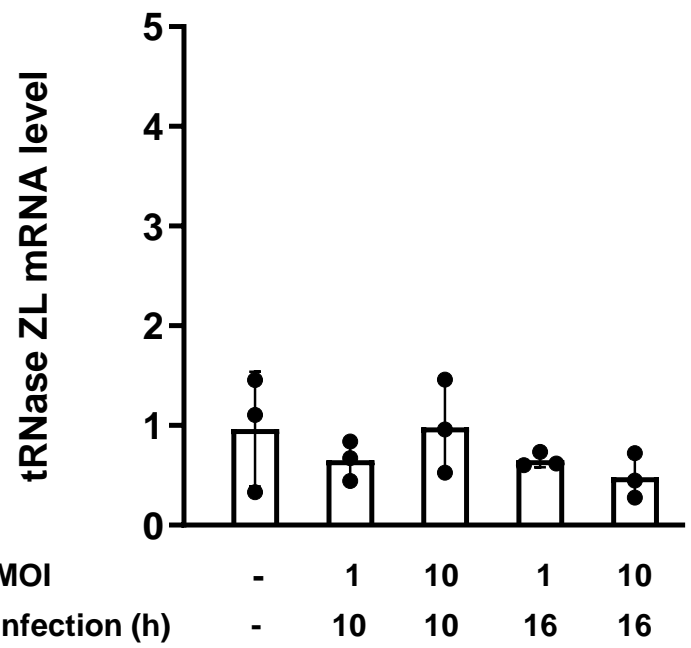

(B)

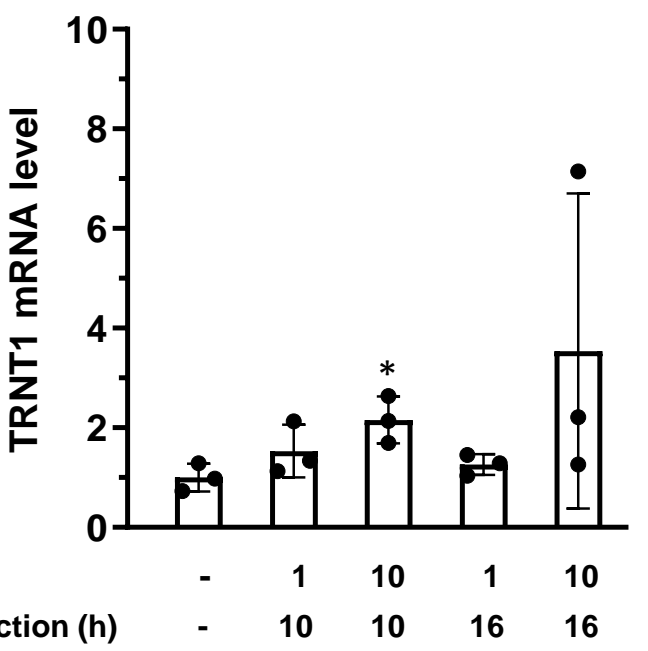

(D)

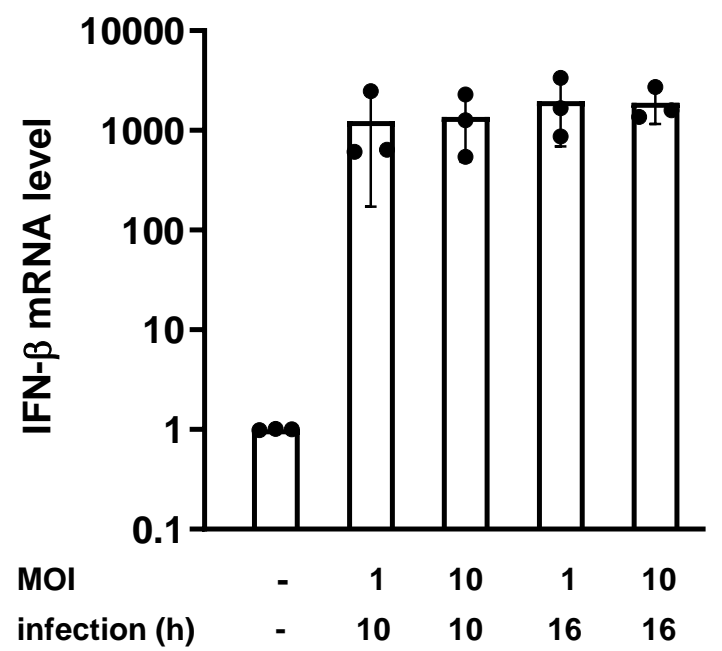


Fig. 3

(A)

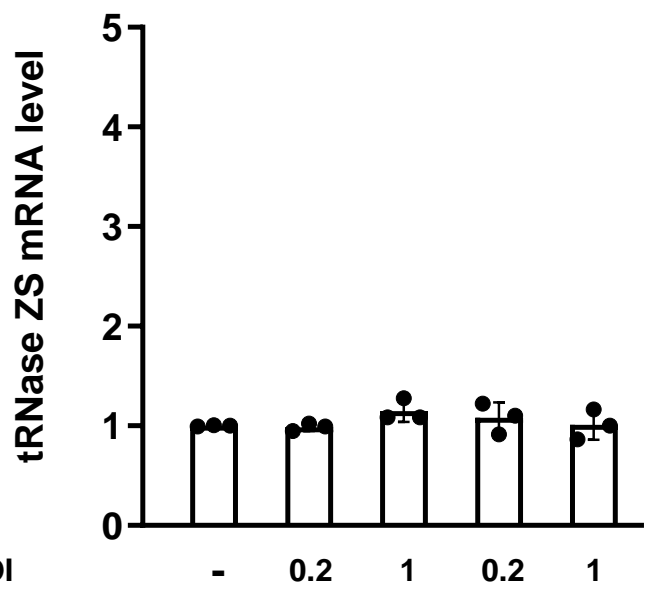

(B)

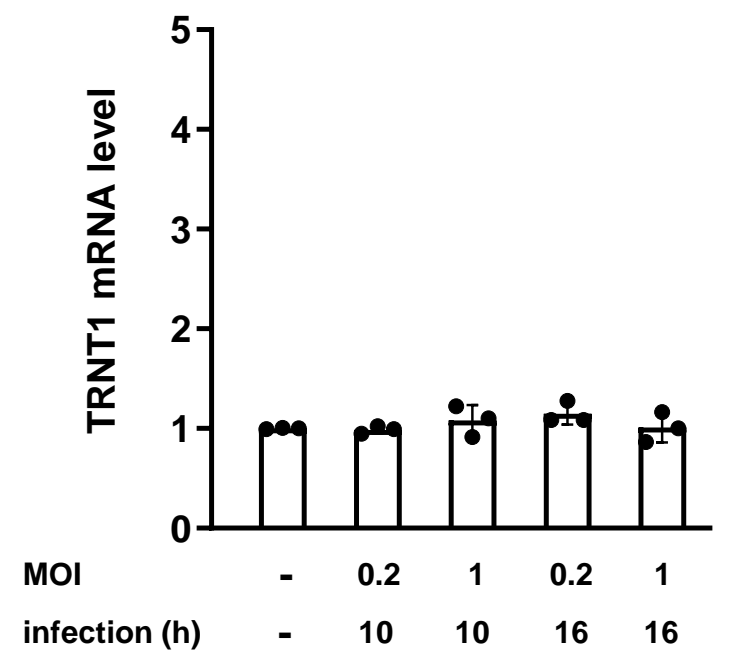

(C)

(D)
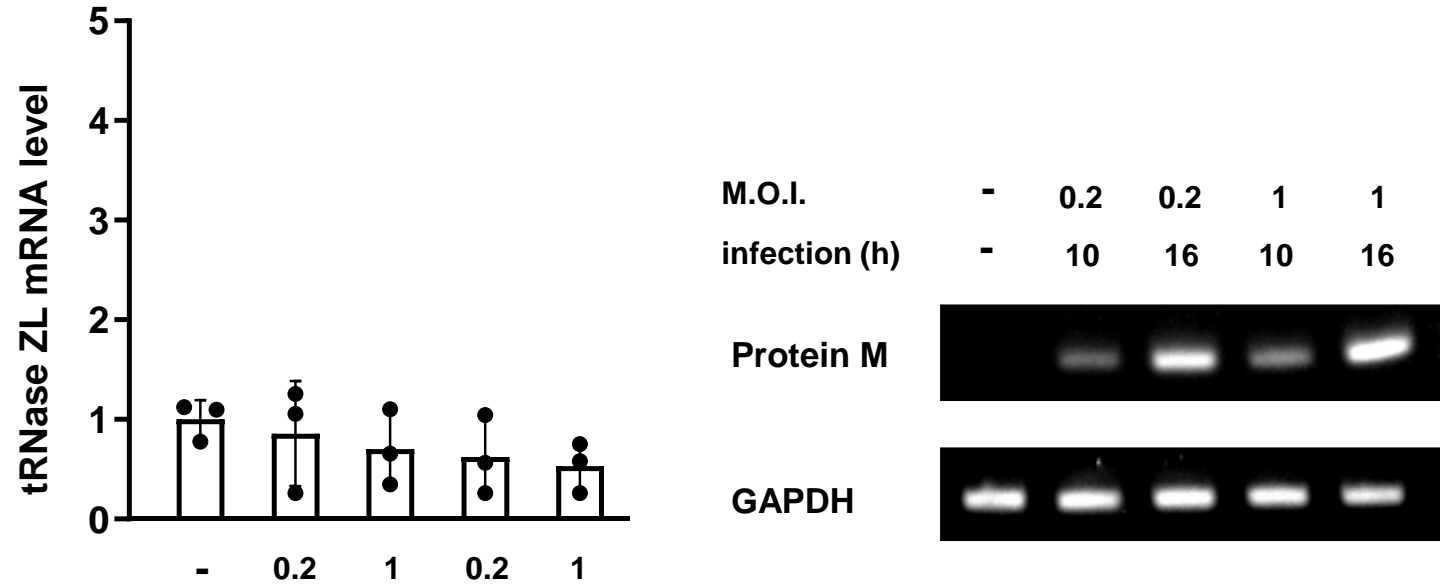

GAPDH

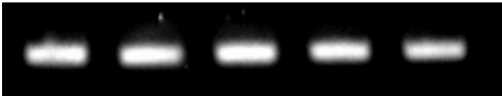

MOI

$\begin{array}{llll}- & 10 \quad 10 \quad 1616\end{array}$ 
Fig. 4

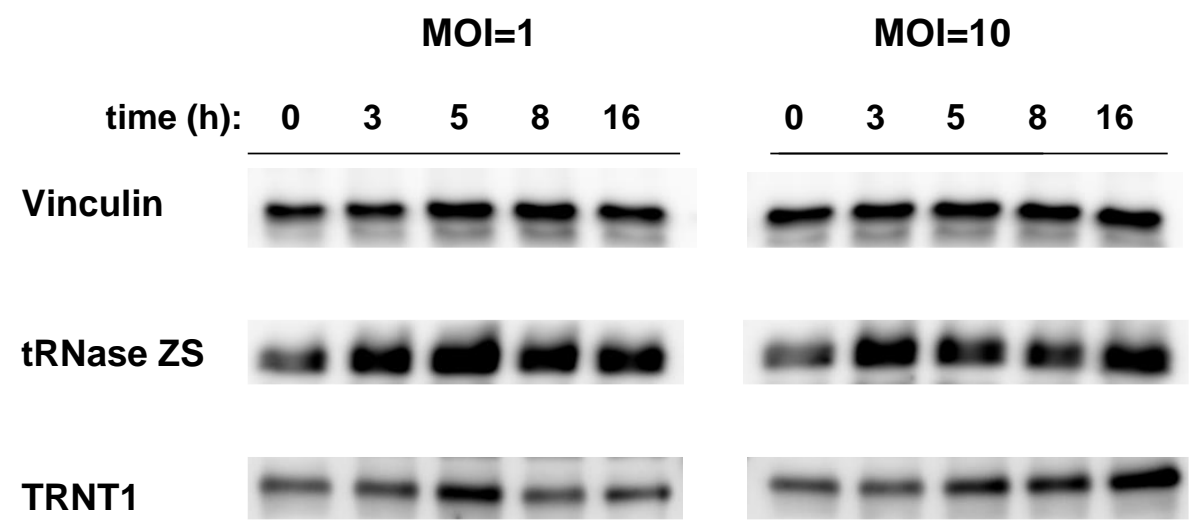

